\title{
COLLECTIVE DYNAMICS OF A DOMAIN WALL - AN OUTLINE
}

\author{
by \\ H. Arodź \\ Institute of Physics, Jagellonian University, \\ Reymonta 4, 30-059 Cracow, Poland
}

We shall consider domain walls in a relativistic field-theoretical model defined by the following Lagrangian

$$
\mathcal{L}=-\frac{1}{2} \eta_{\mu \nu} \partial^{\mu} \Phi \partial^{\nu} \Phi-\frac{\lambda}{2}\left(\Phi^{2}-\frac{M^{2}}{4 \lambda}\right)^{2}
$$

where $\Phi$ is a single real scalar field, $\left(\eta_{\mu \nu}\right)=\operatorname{diag}(-1,1,1,1)$ is the metric in Minkowski space-time, and $\lambda, M$ are positive parameters.

Euler-Lagrange equation corresponding to Lagrangian (1) has the particular time-independent solution

$$
\Phi\left(x^{\mu}\right)=\Phi_{0} \tanh \left(\frac{x^{3}}{2 l_{0}}\right)
$$

which describes a static, planar domain wall stretched along the $x^{3}=0$ plane. Here $\Phi_{0}=M /(2 \sqrt{\lambda})$ denotes one of the two vacuum values of the field $\Phi$ the other one is equal to $-\Phi_{0}$. The parameter $M$ can be identified with the mass of the scalar particle related to the field $\Phi$. The corresponding Compton length $l_{0}=M^{-1}$ gives the physical length scale in the model. Energy density for the planar domain wall (2) is exponentially localised in a vicinity of the $x^{3}=0$ plane. The transverse width of the domain wall is of the order $2 l_{0}$.

The issue is time evolution of a non-planar domain wall. Such domain walls can be infinite, consider, e.g., locally deformed planar domain wall or a cylindrical domain wall. They can also be finite closed, e.g., like a sphere or a torus. We shall restrict our considerations to a single large, smooth 
domain wall. Such a domain wall is defined by a set of conditions which provide Lorentz and reparametrisation invariant formulation of the heuristic requirement that $l_{0}^{2} R_{1,2} \ll 1$ at each point of the domain wall, where $R_{1}, R_{2}$ denote local main curvature radia in a local rest frame of the considered infinitesimal piece of the domain wall. In this case it is possible to develop the presented below perturbative approach to the dynamics of the domain wall. In other cases, e.g. when $l_{0} / R_{i} \sim 1$ at certain points of the domain wall, the only practical tool is numerical analysis.

Physical idea underlying the expansion in the width is rather simple. The domain wall is a stable solitonic object. One expects that each piece of the large, smooth domain wall in the local co-moving reference frame does not differ much from the planar domain wall. The differences, which are due to curvature of the domain wall, can be calculated perturbatively.

The tricky point is to find a convenient reference frame co-moving with the domain wall. From the seminal papers [1, 2], which were devoted to dynamics of vortices but this does not make an essential difference here, we know that the co-moving reference frame should be based on a relativistic membrane. In the leading approximation [1, 2] the membrane is of NambuGoto type, and at each instant of time it coincides with the surface on which the scalar field $\Phi$ vanishes. In the following we will call this surface the core of the domain wall. For instance, in the case of the static domain wall (2) the core is given by the plane $x^{3}=0$. When calculating corrections to the leading approximation one can still adhere to this identification of the membrane with the core, see, e.g., papers [3, 4, 5]. The price for this is that the membrane is no longer of the Nambu-Goto type. Effective action for such membrane contains higher derivatives with respect to time, and probably is nonlocal [6]. The reason for these unpleasant features is that the points at which the scalar field vanishes do not constitute a physical object (the physical object is the domain wall itself and not the core), and therefore the core, being a purely mathematical construct, can have strange from the physical point of view equation of motion. In papers [7, 8] we have proposed to use a membrane which is of Nambu-Goto type to all orders. This membrane coincides with the core only at the initial instant of time.

Our description of the dynamics of the domain wall [7, 8] involves the Nambu-Goto membrane and certain additional 2+1-dimensional fields defined on the membrane. They obey nonlinear equations of motion which are second order partial differential equations, and they describe time evolution 
of the domain wall completely. Transverse profile of the domain wall, that is dependence of the scalar field $\Phi$ on a variable changing in the direction perpendicular to the domain wall, is uniquely and explicitly given once the evolution of the Nambu-Goto membrane and of the 2+1-dimensional fields is found. The dependence on the transverse variable is calculated perturbatively, and one should observe that it is the case of a singular perturbation. Our approach to constructing the perturbative expansion follows a method used in condensed matter physics [9]. Because of the explicit dependence on the transverse variable the dynamics of the domain wall has been reduced to dynamics of the collective degrees of freedom given by the Nambu-Goto membrane and the fields on it. In the following we shall present basic steps of the approach developed in papers [7, 8].

\section{Step 1 . The co-moving coordinate system.}

We introduce a surface $S$ co-moving with the domain wall, that is the comoving membrane. It does not have to coincide with the core, except at the initial instant of time. The world-volume of $S$, denoted by $\Sigma$, is parametrised as follows

$$
\Sigma \ni\left(Y^{\mu}\right)\left(u^{a}\right)=\left(\tau, Y^{i}\left(u^{a}\right)\right) .
$$

We use the notation $\left(u^{a}\right)_{a=0,1,2}=\left(\tau, \sigma^{1}, \sigma^{2}\right)$, where $\tau$ coincides with the laboratory frame time $x^{0}$, while $\sigma^{1}, \sigma^{2}$ parametrise $S$ at each instant of time. The index $i=1,2,3$ refers to the spatial components of the four-vector. The points of the co-moving membrane $S$ at the instant $\tau_{0}$ are given by $\left(Y^{i}\right)\left(\tau_{0}, \sigma^{1}, \sigma^{2}\right)$. The coordinate system $\left(\tau, \sigma^{1}, \sigma^{2}, \xi\right)$ co-moving with the domain wall is defined by the formula

$$
x^{\mu}=Y^{\mu}\left(u^{a}\right)+\xi n^{\mu}\left(u^{a}\right),
$$

where $x^{\mu}$ are Cartesian (laboratory frame) coordinates in Minkowski spacetime, and $\left(n^{\mu}\right)$ is a normalised space-like four-vector orthogonal to $\Sigma$ in the covariant sense,

$$
n_{\mu}\left(u^{a}\right) Y_{, a}^{\mu}\left(u^{a}\right)=0, \quad n_{\mu} n^{\mu}=1,
$$

where $Y_{, a}^{\mu} \equiv \partial Y^{\mu} / \partial u^{a}$. The three four-vectors $Y_{, a}$ are tangent to $\Sigma$. $\xi$ is the transverse variable. Definition (4) implies that $\xi$ and $u^{a}$ are Lorentz scalars. In the co-moving coordinates the co-moving membrane is described by the condition $\xi=0$. For points lying on $S$ the parameter $\tau$ coincides with the laboratory time $x^{0}$, but for $\xi \neq 0$ in general $\tau$ is not equal to $x^{0}$. 
The extrinsic curvature coefficients $K_{a b}$ and induced metrics $g_{a b}$ on $\Sigma$ are defined by the following formulas:

$$
K_{a b}=n_{\mu} Y_{, a b}^{\mu}, \quad g_{a b}=Y_{, a}^{\mu} Y_{\mu, b},
$$

where $a, b=0,1,2$. The covariant metric tensor in the new coordinates has the following form

$$
\left[G_{\alpha \beta}\right]=\left[\begin{array}{ll}
G_{a b} & 0 \\
0 & 1
\end{array}\right],
$$

where $\alpha, \beta=0,1,2,3 ; \quad \alpha=3$ corresponds to the $\xi$ coordinate; and

$$
G_{a b}=N_{a c} g^{c d} N_{d b}, \quad N_{a c}=g_{a c}-\xi K_{a c} .
$$

Thus, $G_{\xi \xi}=1, G_{\xi a}=0$. Straightforward computation gives

$$
\sqrt{-G}=\sqrt{-g} h\left(\xi, u^{a}\right)
$$

where as usual $g=\operatorname{det}\left[g_{a b}\right], \quad G=\operatorname{det}\left[G_{\alpha \beta}\right]$, and

$$
h\left(\xi, u^{a}\right)=1-\xi K_{a}^{a}+\frac{1}{2} \xi^{2}\left(K_{a}^{a} K_{b}^{b}-K_{a}^{b} K_{b}^{a}\right)-\frac{1}{3} \xi^{3} K_{b}^{a} K_{c}^{b} K_{a}^{c} .
$$

For raising and lowering the latin indices of the extrinsic curvature coefficients we use the induced metric tensors $g^{a b}, g_{a b}$.

The inverse metric tensor $G^{\alpha \beta}$ is given by the formula

$$
\left[G^{\alpha \beta}\right]=\left[\begin{array}{ll}
G^{a b} & 0 \\
0 & 1
\end{array}\right]
$$

where

$$
G^{a b}=\left(N^{-1}\right)^{a c} g_{c d}\left(N^{-1}\right)^{d b} .
$$

$N^{-1}$ is just the matrix inverse to $\left[N_{a b}\right]$. It has the upper indices by definition.

In general, the coordinates $\left(u^{a}, \xi\right)$ are defined locally, in a vicinity of the world-volume $\Sigma$ of the membrane. Roughly speaking, the allowed range of the $\xi$ coordinate is determined by the smaller of the two main curvature radia of the membrane in a local rest frame. We assume that this curvature radius is sufficiently large so that on the outside of the region of validity of the comoving coordinates there are only exponential tails of the domain wall, that is that the field $\phi$ is exponentially close to one of the two vacuum solutions. 


\section{Step 2. Field equation in the co-moving coordinates.}

It is convenient to rescale the field $\Phi$ and the coordinate $\xi$,

$$
\Phi\left(x^{\mu}\right)=\frac{M}{2 \sqrt{\lambda}} \phi\left(s, u^{a}\right), \quad \xi=\frac{2}{M} s,
$$

where $\phi$ and $s$ are dimensionless. We also extract from the scalar field its component living on the co-moving membrane and treat it separately from the remaining part of the scalar field. To this end we write the identity

$$
\phi\left(s, u^{a}\right)=B\left(u^{a}\right) \psi(s)+\chi\left(s, u^{a}\right),
$$

where

$$
B\left(u^{a}\right) \stackrel{d f}{=} \phi\left(0, u^{a}\right)
$$

is the component of the scalar field living on the co-moving membrane, and

$$
\chi \stackrel{d f}{=} \phi\left(s, u^{a}\right)-B\left(u^{a}\right) \psi_{0}(s)
$$

is the remaining part. The auxiliary, fixed function $\psi_{0}(s)$ depends on the variable $s$ only. It is smooth, concentrated around $s=0$, and

$$
\psi_{0}(0)=1
$$

It follows that

$$
\chi\left(0, u^{a}\right)=0 .
$$

The best choice for $\psi_{0}(s)$ is given by formula 8

$$
\psi_{0}(s)=\frac{1}{\cosh ^{2}(s)} .
$$

Next we derive Euler-Lagrange equations by taking independent variations of $B\left(u^{a}\right)$ and $\chi$. The variation $\delta \chi$ has to respect condition (9), hence

$$
\delta \chi\left(0, u^{a}\right)=0
$$

Because of this condition, variation of the action functional

$$
\mathcal{S}=\frac{2}{M} \int d s d^{3} u \sqrt{-g} h\left(s, u^{a}\right) \mathcal{L}
$$


with respect to $\chi$ gives Euler-Lagrange equation in the regions $s<0$ and $s>0$. It has the following form

$$
\begin{aligned}
\frac{2}{M^{2}} & \frac{1}{\sqrt{-g}} \partial_{a}\left[\sqrt{-g} h G^{a b} \partial_{b}\left(B \psi_{0}+\chi\right)\right] \\
& +\frac{1}{2} \partial_{s}\left[h \partial_{s}\left(B \psi_{0}+\chi\right)\right]+h\left(B \psi_{0}+\chi\right)\left[1-\left(B \psi_{0}+\chi\right)^{2}\right]=0 .
\end{aligned}
$$

At $s=0$ there is no Euler-Lagrange equation corresponding to the variation $\delta \chi$. Instead, we have the condition (9). Equation (10) should be solved in the both regions separately, with (9) regarded as a part of boundary conditions for $\chi$. To complete the boundary conditions we also specify the behaviour of $\chi$ for $|\xi|$ much larger than the characteristic length $l_{0}$, that is for $|s| \gg 1$ : we shall seek a solution such that $\chi$ is exponentially close to +1 for $s \gg 1$, while for $s \ll-1$ it is exponentially close to -1 .

At this stage of considerations Eq.(10) should not be extrapolated to $s=0$. For example, the 1.h.s. of it could have a $\delta(s)$-type singularity. It would occur if $\chi$ was smooth for $s>0$ and for $s<0$ but had a spike at $s=0$.

In addition to Eq.(10) we also have the Euler-Lagrange equation corresponding to variations of $B\left(u^{a}\right)$. This equation has the following form

$$
\begin{aligned}
& \frac{2}{M^{2}} \int d s \frac{1}{\sqrt{-g}} \partial_{a}\left[\sqrt{-g} h G^{a b} \partial_{b}\left(B \psi_{0}+\chi\right)\right] \psi_{0} \\
& \quad-\frac{1}{2} \int d s h \partial_{s} \psi_{0} \partial_{s}\left(B \psi_{0}+\chi\right) \\
& \quad+\int d s h \psi_{0}\left(B \psi_{0}+\chi\right)\left[1-\left(B \psi_{0}+\chi\right)^{2}\right]=0 .
\end{aligned}
$$

Here and in the following we use $\int d s$ as a shorthand for the definite integral $\int_{-\infty}^{+\infty} d s$.

Using equations (10) and (11) one can prove that $\chi$ does not have the spike at $s=0$. It follows that Eq.(10) is obeyed by $\chi$ also at $s=0$, and that now Eq.(11) can be obtained by multiplying Eq.(10) by $\psi_{0}(s)$ and integrating over $s$. Therefore we may concentrate on solving Eq.(10).

\section{Step 3. Expansion in the width.}

First we solve Eq.(10) in the leading approximation obtained by putting $1 / M=0$. The equation is then reduced to

$$
\frac{1}{2} \partial_{s}^{2} \phi^{(0)}+\phi^{(0)}\left[1-\left(\phi^{(0)}\right)^{2}\right]=0
$$


where

$$
\phi^{(0)}=B^{(0)} \psi_{0}+\chi^{(0)} .
$$

Equation (12) does not contain derivatives with respect to time, in spite of the fact that it is supposed to approximate the evolution equation (10). This annoying fact is due to the singular character of the perturbation given in Eq.(10) by the terms proportional to positive powers of $1 / M$. We shall see that time evolution is obtained indirectly, from consistency conditions.

Equation (12) has the following particular, well-known solution

$$
\phi^{(0)}=\tanh s .
$$

This solution together with conditions (8), (9) gives

$$
B^{(0)}=0, \quad \chi^{(0)}=\tanh s .
$$

Notice that $\phi^{(0)}$ has the same form as the planar domain wall $(2)$ - in this way we realise the idea that in the co-moving reference frame the domain wall does not differ much from the planar domain wall.

The solution (13) in the co-moving coordinates does not determine the field $\phi$ in the laboratory frame because we do not know yet the position of the co-moving membrane with respect to the laboratory frame. Equations (10), (11) yield an equation for the co-moving membrane, otherwise they would not form the complete set of evolution equations for the field $\Phi$. In fact, we shall see that the first order terms in Eq.(10) imply Nambu-Goto equation for the membrane.

The expansion in the width has the form

$$
\begin{gathered}
\chi\left(s, u^{a}\right)=\tanh s+\frac{1}{M} \chi^{(1)}\left(s, u^{a}\right)+\frac{1}{M^{2}} \chi^{(2)}\left(s, u^{a}\right)+\frac{1}{M^{3}} \chi^{(3)}\left(s, u^{a}\right)+\ldots, \\
B\left(u^{a}\right)=\frac{1}{M} B^{(1)}\left(u^{a}\right)+\frac{1}{M^{2}} B^{(2)}\left(u^{a}\right)+\frac{1}{M^{3}} B^{(3)}\left(u^{a}\right)+\ldots
\end{gathered}
$$

where we have taken into account the zeroth order results (14). The expansion parameter is $1 / M$ and not $1 / M^{2}$ because $1 / M$ in the first power appears in $h$ and $G^{a b}$ functions after passing to the $s$ variable. In order to obey the condition (9), and to ensure the proper asymptotics of $\chi$ at large $|s|$ we assume that for $n \geq 1$

$$
\chi^{(n)}\left(0, u^{a}\right)=0, \quad \lim _{s \rightarrow \pm \infty} \chi^{(n)}=0 .
$$


Inserting the perturbative Ansatz $(15,16)$ in Eqs.(10, 11), expanding the l.h.s.'s of them in powers of $1 / M$, and equating to zero coefficients in front of the powers of $1 / \mathrm{M}$ we obtain a sequence of linear, inhomogeneous equations for $\chi^{(n)}\left(s, u^{a}\right), B^{(n)}\left(u^{a}\right)$ with $n \geq 1$.

In particular, Eq.(10) expanded in the powers of $1 / M$ gives equations of the type

$$
\hat{L} \chi^{(n)}=f^{(n)},
$$

where the source term $f^{(n)}$ is determined by the lower order terms in $\chi$ and $B$, and

$$
\hat{L} \stackrel{d f}{=} \frac{1}{2} \partial_{s}^{2}+1-3\left(\chi^{(0)}\right)^{2} .
$$

Explicit solution of Eq.(18) is given by the formula

$$
\chi^{(n)}(s)=2 \psi_{1}(s) \int_{-\infty}^{s} d x \psi_{0}(x) f^{(n)}(x)-2 \psi_{0}(s) \int_{0}^{s} d x \psi_{1}(x) f^{(n)}(x) .
$$

This solution obeys the boundary conditions (17).

Obviously, we assume that all proportional to positive powers of $1 / M$ terms in Eqs. $(10,11)$ are small. For this it is not sufficient that the extrinsic curvatures are small, that is that $l_{0} K_{b}^{a} \ll 1$. We have also to assume that the derivatives $\chi_{, a}^{(n)}, B_{, a}^{(n)}$ are not proportional to $\mathrm{M}$. It is not the case, for example, if $\chi$ and $B$ contain modes oscillating with a frequency $\sim M$. They would give positive powers of $M$ upon differentiation with respect to $u^{a}$. If such oscillating components were present the counting of powers of $1 / M$ would no longer be so straightforward as we have assumed. This assumption excludes radiation modes as well as massive excitations of the domain wall. Therefore, the approximate solution we obtain gives what we may call the basic curved domain wall. To obtain more general domain wall solutions one would have to generalize appropriately the approximation scheme. Actually, the fact that such particular radiationless, unexcited curved domain wall exists is a prediction coming from the $1 / M$ expansion. The expansion yields domain walls of concrete transverse profile - the dependence on $s$ is explicit in the approximate solution we construct even at the initial instant of time. We may choose the initial position and velocity of points of the membrane but the dependence of the scalar field on the variable $s$ at the initial time is given by formulas (19). This unique profile is characteristic for the basic curved domain wall. 


\section{Step 4. The consistency conditions.}

The first order terms in Eq.(10) give the following equation

$$
\hat{L} \chi^{(1)}=K_{a}^{a} \partial_{s} \chi^{(0)},
$$

where $\chi^{(0)}$ is given by the second of formulas (14).

The most important point in our approach is the observation that operator $\hat{L}$ has a zero-mode, that is the normalizable solution

$$
\psi_{0}(s)=\frac{1}{\cosh ^{2} s}
$$

of the homogeneous equation

$$
\hat{L} \psi_{0}=0
$$

Notice that $\psi_{0}=\partial_{s} \chi^{(0)}$ — this means that the zero-mode $\psi_{0}$ is related to the translational invariance of Eq.(12) under $s \rightarrow s+$ const. The presence of the zero-mode implies the consistency conditions. For example, let us multiply Eq. (20) by $\psi_{0}$ and integrate over $s$. It is easy to see that $\int \psi_{0} \hat{L} \chi^{(1)}$ vanishes because of (21), and we obtain the following condition

$$
K_{a}^{a} \int d s \psi_{0}(s) \partial_{s} \chi^{(0)}(s)=0
$$

which is equivalent to

$$
K_{a}^{a}=0 .
$$

Eq.(22) coincides with the well-known Nambu-Goto equation. It determines the motion of the co-moving membrane, that is the functions $Y^{i}\left(u^{a}\right), \mathrm{i}=1,2,3$, once initial data are fixed. When we know these functions we can calculate the extrinsic curvature coefficients $K_{a b}$ and the metric $g_{a b}$. Review of properties of relativistic Nambu-Goto membranes can be found in, e.g., [9].

Due to Nambu-Goto equation (22) the r.h.s. of Eq.(20) vanishes and the resulting homogeneous equation

$$
\hat{L} \chi^{(1)}=0
$$

with the boundary conditions (17) has only the trivial solution

$$
\chi^{(1)}=0 .
$$


Notice that vanishing $\chi^{(1)}$ does not mean that the first order correction to the total field $\phi$ also vanishes - there is the first order contribution equal to $B^{(1)} \psi_{0} / M$. It does not vanish on the co-moving membrane that is at $s=0$.

Analogous reasoning can give nontrivial consistency conditions also for equations (18) with $n>1$. For some $n$, e.g. $n=2$ we obtain only the trivial identity $0=0$.

Equation (11) expanded in powers of $1 / M$ gives equations for $B^{(n)}$ coinciding with the consistency conditions. This follows from the fact that both Eq.(11) and the consistency conditions are obtained by multiplying Eq.(10) by the zero-mode $\psi_{0}$ and next integrating over $s$. Euler-Lagrange equation (11) can be regarded as generating equation for the consistency conditions.

Equations (10), (11) in the first order do not give any restriction on the function $B^{(1)}$. Equation for $B^{(1)}$ follows from the third order terms in Eq.(11):

$\frac{1}{\sqrt{-g}} \partial_{a}\left(\sqrt{-g} g^{a b} \partial_{b} B^{(1)}\right)+\left(\frac{\pi^{2}}{4}-1\right) K_{b}^{a} K_{a}^{b} B^{(1)}+\frac{9}{35}\left(B^{(1)}\right)^{3}=\left(\frac{\pi^{2}}{6}-1\right) K_{b}^{a} K_{c}^{b} K_{a}^{c}$,

This situation is typical for singular perturbation theories of which the $1 / M$ expansion is an example - higher order equations imply restrictions (the consistency conditions) for the lower order contributions [10].

\section{Step 5. Initial data.}

At this point we have the complete set of equations determining the evolution of the domain wall in the $1 / M$ expansion. Each of Eqs.(10), (11), (22) describes different aspect of the dynamics of the curved domain wall. Expanded in the positive powers of $1 / M$ Eq.(10) determines dependence of $\chi$ on $s$. Because the term $B \psi_{0}$ in formula (5) has explicit dependence on $s$, we may say that Eq.(10) for $\chi$ fixes the transverse profile of the domain wall.

Equation (11) determines the $B^{(n)}\left(u^{a}\right)$ functions, which can be regarded as $(2+1)$-dimensional scalar fields defined on $\Sigma$ and having nontrivial nonlinear dynamics. The extrinsic curvature $K_{a b}$ of $\Sigma$ acts as an external source for these fields. The fields $B^{(n)}$ can propagate along $\Sigma$. One may regard this effect as causal propagation of deformations which are introduced by the extrinsic curvature.

Finally, Nambu-Goto equation (22) for the co-moving membrane determines the evolution of the shape of the domain wall.

Equation (22) for the co-moving membrane and equations for $B^{(n)}$ obtained from the consistency conditions are of the evolution type - we have 
to specify initial data for them, otherwise their solutions are not unique. Equations (18) for the perturbative contributions $\chi^{(n)}$ are of different type - in order to ensure uniqueness of their solution it is sufficient to adopt the boundary conditions (17). The initial data for $B\left(u^{a}\right)$ and $Y^{i}\left(u^{a}\right)$ follow from initial data for the original field $\phi$. From such data for $\phi$ we know the initial position and velocity of the core. We assume that at the initial instant $\tau_{0}$ the co-moving membrane and the core have the same position and velocity. Hence,

initial data for the membrane $=$ initial data for the core.

Using formula (5) one can show that then

$$
B^{(n)}\left(\tau_{0}, \sigma^{1}, \sigma^{2}\right)=0, \quad \partial_{\tau} B^{(n)}\left(\tau_{0}, \sigma^{1}, \sigma^{2}\right)=0 .
$$

In order to find the domain wall solution one should first solve the collective dynamics, that is to compute evolution of the co-moving membrane and of the $B^{(n)}$ fields. The profile $\chi$ of the domain wall is found in the next step from formulas $(15,19)$. In our perturbative scheme the profile of the domain wall can not be chosen arbitrarily even at the initial time - it is fixed uniquely once the initial data for the membrane and for the $B$ field are given. Evolution of the core can be determined afterwards, from the explicit expression for the scalar field $\phi[8]$.

$$
* * *
$$

In paper [8] the perturbative solution has been constructed up to the fourth order.

In paper 111 second order perturbative solutions for cylindrical and spherical domain walls have been compared with numerical solutions of the EulerLagrange equation. The results of the comparison are quite encouraging.

In paper [12] analogous approach has been applied to a vortex in the Abelian Higgs model.

The perturbative approach to dynamics of the domain walls we have sketched can be generalised to models involving several fields. Also the requirement of relativistic invariance can be dropped out. 
Acknowledgment. It is a pleasure to thank the organizers for nice and stimulating atmosphere during the Workshop.

\section{References}

[1] H.B.Nielsen and P.Olesen, Nucl.Phys. B61, 45 (1973).

[2] D.Förster, Nucl.Phys. B81, 84 (1974).

[3] R.Gregory, Phys.Lett.B206, 199 (1988).

[4] S.M.Barr and D.Hochberg, Phys.Rev.D39, 2308 (1989).

[5] B.Carter and R.Gregory, Phys. Rev. D51, 5839 (1995).

[6] H.Arodź and P.Wȩgrzyn, Phys.Lett.B291, 251 (1992).

[7] H. Arodź, Nucl. Phys. B450, 174 (1995).

[8] H. Arodź, Nucl. Phys. B, in press (hep-th/9703168).

[9] B. Carter, in "Formation and Interactions of Topological Defects", p.303. A.-Ch. Davis and R. Brandenberger (Eds.). Plenum Press, New York and London, 1995.

[10] N.G.van Kampen, Stochastic Processes in Physics and Chemistry. North-Holland Publ.Comp., Amsterdam, 1987. Chapt.8,§7.

[11] J. Karkowski and Z. Świerczyński, Acta Phys. Pol. B 무, 234 (1996).

[12] H. Arodź, Nucl. Phys. B450, 189 (1995). 